\title{
The continuous wavelet transform in the extraction of information from wavefields
}

Juan Guillermo Paniagua, Instituto Tecnológico Metropolitano, Olga Lucía Quintero, Universidad EAFIT

Copyright 2019, SBGf - Sociedade Brasileira de Geofísica

This paper was prepared for presentation during the $16^{\text {th }}$ International Congress of the Brazilian Geophysical Society held in Rio de Janeiro, Brazil, 19-22 August 2019.

Contents of this paper were reviewed by the Technical Committee of the $16^{\text {th }}$ International Congress of the Brazilian Geophysical Society and do not necessarily represent any position of the SBGf, its officers or members. Electronic reproduction or storage of any part of this paper for commercial purposes without the written consent of the Brazilian Geophysical Society is prohibited.

\section{Abstract}

Artifacts in reverse time migration (RTM) are low spatial frequency noise that appears due to the undesired correlation of head waves, diving waves and backscattered waves in seismic migrated images when the zero-lag cross-correlation imaging condition is applied. Effects associated with artifacts are the possible concealment of important details in images. Different methods have been proposed to eliminate or attenuate these artifacts by modifications of the wave equation, using different imaging conditions or filtering techniques.

In this work we propose the use of the continuous wavelet transform to extract relevant information about source and receiver wavefields in order to obtain data of components of these wavefields.

\section{Introduction}

Reverse time migration (RTM) solves the two-way acoustic wave equation, by the propagation in time domain of the source wavefield in forward direction, and of the receiver wavefield in backward direction. The scalar field (image) is obtained by a mathematical relationship between the extrapolated source and receiver wavefields summed over the sources or so many others, it is known in geophysics as imaging condition and the commonly used is the zerolag cross-correlation.

Then, the conventional zero-lag cross-correlation imaging condition is given by (CLAERBOUT, 1971, 1985)

$$
I(\boldsymbol{x})=\sum_{j}^{s_{\max }} \sum_{i=1}^{t_{\max }} S\left(\boldsymbol{x} ; t_{i} ; s_{j}\right) R\left(\boldsymbol{x} ; t_{i} ; s_{j}\right)
$$

where $x=(x, z)$ is the location in the Cartesian coordinate system, $S\left(\boldsymbol{x} ; t_{i} ; s_{j}\right)$ and $R\left(\boldsymbol{x} ; t_{i} ; s_{j}\right)$ are source and receiver wavefields, respectively, $t_{\max }$ is the total time, $s_{\max }$ is the total number of sources, and $I$ is the final RTM image.

It produces kinematically correct images of the geometry of the subsurface structure (CHATTOPADHYAY and MCMECHAN, 2008). The final image is kinematically accurate at the reflectors due to the fact that incident and reflected wavefields are coincident in space and time (LIU et al., 2011), but the migrated amplitudes no longer hold any physical meaning.
It has been demonstrated that the zero-lag crosscorrelation imaging condition is computationally simpler to implement and favorable than another mathematical expression used to obtain the final image.

However, the final image is contaminated with low spatial frequency noises which can affect its interpretation. These low spatial frequency noises are called artifacts.

Such artifacts result from the unwanted cross-correlation of source and receiver wavefields at non-reflecting points along the raypath. This type of noises is unique in reverse time migration: They are not present in one-way equationbased migration images constructed using the same crosscorrelation imaging condition.

Several works have been developed around to attenuate these low-frequency artifacts, preserving reflections, and improving the model quality, implementing other strategies such as modifications of the wave equation (LOEWENTHAL et al, 1987, BAYSAL et al., 1984), proposing other imaging conditions (GUITTON et al., 2007, VALENCIANO \& BIONDI, 2003, KAELIN \& GUITTON, 2006, PESTANA et al., 2014, SHRAGGE, 2014) and using image filtering techniques (YOUN \& ZHOU, 2001, GUITTON et al., 2006, PANIAGUA et al., 2017).

One strategy to avoid artifacts in RTM is the wavefield decomposition (FEI \& LUO, 2010, LIU et al., 2011, CHEN \& HE, 2014). In this method, source and receiver wavefields are decomposed in their one-way components along a certain specific direction in order to correlate appropriate combinations of some of these decomposed wavefields. When components of source and receiver wavefields that propagate in opposite directions are correlated, it produces a model of the subsurface structures. The cross-correlation of decomposed source and receiver wavefields that propagate in parallel directions will not generate an image because one of them will be zero.

To avoid low-frequency artifacts, Fei et al. (2010) proposed a De-blending RTM algorithm to decompose source and receiver wavefields into upgoing and downgoing wavefields and compare images obtained by crosscorrelation of different combinations of these wavefields.

Liu et al. (2011) proposed the wavefield separation crosscorrelation imaging condition to suppress artifacts. In this method, source and receiver wavefields are decomposed in upgoing and downgoing directions using the 2D Fourier transform. Chen and $\mathrm{He}$ (2014) used the Poynting vector to the separate wavefields in up-going, down-going, leftgoing, and right-going waves and applied the normalized wavefield separation cross-correlation imaging condition.

we propose a way to extract information from source and receiver wavefields based on the analysis of the time-scale 
or time-frequency characteristics, using the continuous wavelet transform (CWT) (PANIAGUA, 2018). The aim is to reduce the low frequency noise in images obtained through RTM and the zero-lag cross-correlation imaging condition correlating some specific information of components of both wavefields.

\section{Method}

Reverse time migration can be represented mathematically by the integral equation (SCHUSTER, 2010):

$$
\begin{aligned}
& m(\boldsymbol{X})=\int_{D}\left[g(\boldsymbol{g},-t \mid \boldsymbol{X}, 0) * d\left(\boldsymbol{g}, t \mid \boldsymbol{X}_{\boldsymbol{s}}, 0\right)\right] \otimes[f(t) \\
& \left.* g\left(\boldsymbol{X}, t \mid \boldsymbol{X}_{\boldsymbol{s}}, 0\right)\right]\left.\right|_{t=0} d g
\end{aligned}
$$

where $\boldsymbol{X}=(x, y, z)$ is the location in the Cartesian coordinate system in the semi-infinite domain $\mathbf{D}$, where $z>$ $0 ; f(t)$ is the source wavelet function; $g\left(\boldsymbol{X}, t \mid \boldsymbol{X}_{\boldsymbol{s}}, 0\right)$ is the acausal Green's function; $d\left(\boldsymbol{g}, t \mid \boldsymbol{X}_{s}, 0\right)$ is the shot gather traces; $g(\boldsymbol{g},-t \mid \boldsymbol{X}, 0) * d\left(\boldsymbol{g}, t \mid \boldsymbol{X}_{\boldsymbol{s}}, 0\right)$ is the back propagated field to be called "the receiver wavefield", $R\left(\boldsymbol{X}, \boldsymbol{X}_{\boldsymbol{s}}, t\right) ; f(t) *$ $g\left(\boldsymbol{X}, t \mid \boldsymbol{X}_{\boldsymbol{s}}, 0\right)$ is the forward propagated source field for a single source at $\boldsymbol{X}_{\boldsymbol{s}}$ to be called "the source wavefield", $S\left(\boldsymbol{X}, \boldsymbol{X}_{\boldsymbol{s}}, t\right)$; and $m(\boldsymbol{X})$ is the RTM migration model. Symbol $\otimes$ represents the temporal dot product.

From eq. (2), let $S(\boldsymbol{X}, t)$ and $R(\boldsymbol{X}, t)$ be source and receiver wavefields, respectively. $S(\boldsymbol{X}, t)$ and $R(\boldsymbol{X}, t)$ can be partitioned mathematically as

$$
S(\boldsymbol{X}, t)=S_{d}(\boldsymbol{X}, t)+S_{u}(\boldsymbol{X}, t)
$$

$S_{d}(\boldsymbol{X}, t)$ and $S_{u}(\boldsymbol{X}, t)$ are subsets of $S(\boldsymbol{X}, t)$, called downgoing and upgoing source wavefields, respectively.

And

$$
R(\boldsymbol{X}, t)=R_{d}(\boldsymbol{X}, t)+R_{u}(\boldsymbol{X}, t)
$$

$R_{d}(\boldsymbol{X}, t)$ and $R_{u}(\boldsymbol{X}, t)$ are subsets of $R(\boldsymbol{X}, t)$, called downgoing and upgoing receiver wavefields, respectively.

Taking into account eq. (1) and replacing eq. (3) and eq. (4), the zero-lag cross-correlation imaging condition can be expressed as follows

$$
\begin{aligned}
I(\boldsymbol{X})=\sum_{j}^{s_{\max }} \sum_{i=1}^{t_{\max }}[ & S_{d}\left(\boldsymbol{X} ; t_{i} ; s_{j}\right) R_{u}\left(\boldsymbol{X} ; t_{i} ; s_{j}\right) \\
& +S_{u}\left(\boldsymbol{X} ; t_{i} ; s_{j}\right) R_{d}\left(\boldsymbol{X} ; t_{i} ; s_{j}\right) \\
& +S_{d}\left(\boldsymbol{X} ; t_{i} ; s_{j}\right) R_{d}\left(\boldsymbol{X} ; t_{i} ; s_{j}\right) \\
& \left.+S_{u}\left(\boldsymbol{X} ; t_{i} ; s_{j}\right) R_{u}\left(\boldsymbol{X} ; t_{i} ; s_{j}\right)\right]
\end{aligned}
$$

Then,

$$
I(\boldsymbol{X})=I_{d u}(\boldsymbol{X})+I_{u d}(\boldsymbol{X})+I_{d d}(\boldsymbol{X})+I_{u u}(\boldsymbol{X})
$$

Based on Fei \& Luo, (2010) and Liu et al., (2011), $I_{d d}(\boldsymbol{X})$ and $I_{u u}(\boldsymbol{X})$ are the cross-correlation of two wavefields that propagate in the same direction downgoing $\left(I_{d d}\right)$ or upgoing $\left(I_{u u}\right)$ and generate artifacts in the RTM image.

Thus, the wavefield decomposition cross-correlation imaging condition can be formulated by keeping only the first two terms as follows

$$
\begin{aligned}
I(\boldsymbol{X})=\sum_{j}^{s_{\max }} \sum_{i=1}^{t_{\max }}\left[S_{d}\left(\boldsymbol{X} ; t_{i} ; s_{j}\right) R_{u}\left(\boldsymbol{X} ; t_{i} ; s_{j}\right)\right. \\
\left.+S_{u}\left(\boldsymbol{X} ; t_{i} ; s_{j}\right) R_{d}\left(\boldsymbol{X} ; t_{i} ; s_{j}\right)\right]
\end{aligned}
$$

From eq. (7), we have

$$
I_{d u}(\boldsymbol{X})=\sum_{j}^{s_{\max }} \sum_{i=1}^{t_{\max }} S_{d}\left(\boldsymbol{X} ; t_{i} ; s_{j}\right) R_{u}\left(\boldsymbol{X} ; t_{i} ; s_{j}\right)
$$

which is the cross-correlation of downgoing source and upgoing receiver wavefields that is exactly what one will get in one-way wave-equation migration.

\section{Extraction of information of wavefields}

Let $S(x, z, t)$ and $R(x, z, t)$ be source and receiver wavefields. We apply the CWT to source and receiver wavefields for a fixed value in $x$-axis, that is, we use subsets $S\left(x_{i}, z, t\right)$ which will be denoted by $S_{x_{i}}(z, t)$ and $R\left(x_{i}, z, t\right)$ which will be denoted by $R_{x_{i}}(z, t)$ of source and receiver wavefields for a fixed $x=x_{i}$ value. For each value $x=x_{i}$, wavefields $S_{x_{i}}(z, t)$ and $R_{x_{i}}(z, t)$ are analyzed by applying the one dimensional continuous wavelet transform (1D-CWT), and we find a similar structure in subsets of the source wavefield and of the receiver wavefield. We find some common characteristics in coefficients obtained by 1D-CWT and in components of wavefields.

\section{Source wavefield $S(x, z, t)$}

We apply the 1D-CWT on the subset $S_{x_{i}}(z, t)$ with a fixed value of $z=z_{i}$, denoted by $S_{x_{i}, z_{i}}(t)$, to obtain the scalogram of the signal. That is, for a fixed $x=x_{i}$ and $z=$ $z_{i}, S_{x_{i}, z_{i}}(t)=S\left(x_{i}, z_{i}, t\right)$, by 1D-CWT of $S_{x_{i}, z_{i}}(t)$ we obtain the field $\hat{S}_{x_{i}, z_{i}}(u, s)=0 W\left(S_{x_{i}, z_{i}}(t)\right)$ and the scalogram of the signal is obtained. $\hat{S}_{x_{i}, z_{i}}(u, s)$ is the 1D-CWT of $S_{x_{i}, z_{i}}(t)$.

Analyzing the obtained scalograms, the minimum value of all coefficients corresponds to the downgoing wavefield. This coefficient is selected from $\hat{S}_{x_{i}, z_{i}}(u, s)$ and located in the time signal $S_{x_{i}, z_{i}}(t)$ considering the delay of the signal in time. This point is saved in a new wavefield $S_{N}(x, z, t)$. To improve the accuracy, two more points are taken before and after this point and saved in $S_{N}(x, z, t)$ too. This process is done until you get the whole wavefield $S_{N}(x, z, t)$. This is the source wavefield of the incident wave (Downgoing source wavefield).

\section{Receiver wavefield $R(x, z, t)$}

We apply the 1D-CWT on the subset $R_{x_{i}}(z, t)$ with a fixed value $t=t_{i}$, denoted by $R_{x_{i}, t_{i}}(z)$, to obtain the scalogram of the signal. That is, for a fixed $x=x_{i}$ and $t=t_{i}$, $R_{x_{i}, t_{i}}(z)=R\left(x_{i}, z, t_{i}\right)$, by 1D-CWT of $R_{x_{i}, t_{i}}(z)$ we obtain the field $\hat{R}_{x_{i}, t_{i}}(u, s)=W\left(R_{x_{i}, t_{i}}(z)\right)$ and the scalogram of the signal is obtained. $\hat{R}_{x_{i}, t_{i}}(u, s)$ is the 1D-CWT of $R_{x_{i}, t z_{i}}(z)$.

Analyzing the obtained scalograms, the maximum absolute value of coefficients corresponds to a coefficient with negative value. This coefficient is selected and located 
in the time signal $R_{x_{i}, t_{i}}(z)$ taking into account the delay of the signal in time. This point is saved in a new wavefield $R_{N}(x, z, t)$. To improve the accuracy, two more points are taken before and after this point and saved in $R_{N}(x, z, t)$ too. This process is repeated until you get the whole wavefield $R_{N}(x, z, t)$. This is the receiver wavefield of the incident wave (Upgoing receiver wavefield).

With wavefields obtained by using this methodology $S_{N}(x, z, t)$ and $R_{N}(x, z, t)$, we use eq. (8) to obtain the migrated image.

\section{Numerical examples}

We apply the RTM algorithm using a velocity model depicted in Figure 1. We use only one source point located at $X=1.5 \mathrm{Km}$ from the beginning of the surface. There are 400 receivers equally distributed along the surface and the receiver interval is $7.5 \mathrm{~m}$.

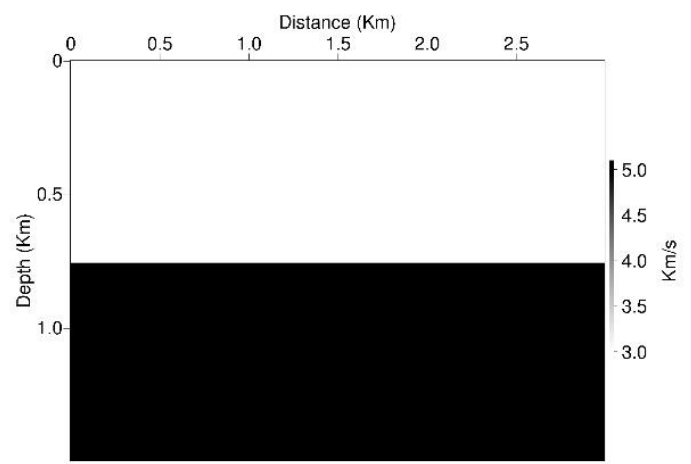

Figure 1. Two-layer velocity model

The migrated model obtained by RTM algorithm and the conventional zero-lag cross-correlation imaging condition is shown in Figure 2.

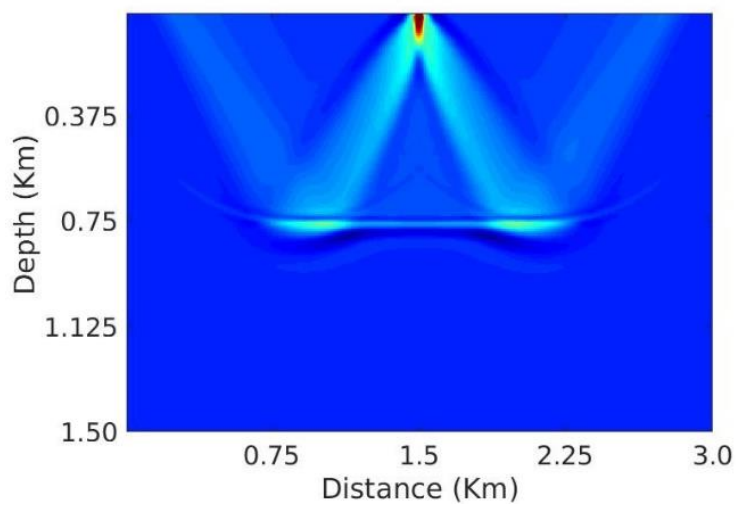

Figure 2. Migrated image obtained by RTM and the zerolag cross-correlation imaging condition.

We can see that the migrated image is contaminated with low frequency artifacts above and near the reflective event and in the shallow parts.

Following the proposed methodology to analyze the source wavefield, with a fixed value of $x=x_{i}$, the $S_{x_{i}}(z, t)$ wavefield is obtained from the $S(x, z, t)$ wavefield. Figure 3 shows the $S_{x_{i}}(z, t)$ wavefield from an arbitrarily chosen value of $x$.

For each value in $z=z_{i}$ axis, the 1D-CWT is performed on the $S_{x_{i}, z_{i}}(t)$ wavefield. Figure $\left.3 a\right)$ shows a $S_{x_{i}}(z, t)$ wavefield at $x=1.125 \mathrm{Km}$ and, Figure $3 \mathrm{~b}$ ) and Figure $3 \mathrm{c}$ ) show the signal $S_{x_{i}, z_{i}}(t)$ at $z=0.45 \mathrm{Km}$ and its corresponding coefficients obtained by CWT of $S_{x_{i}, z_{i}}(t)$.

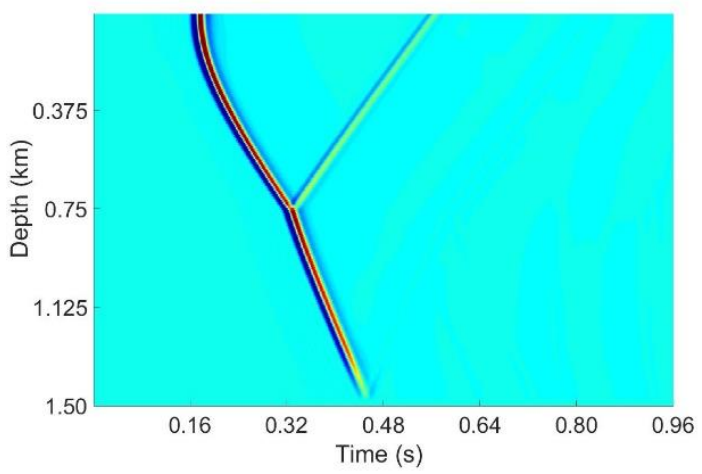

(a)

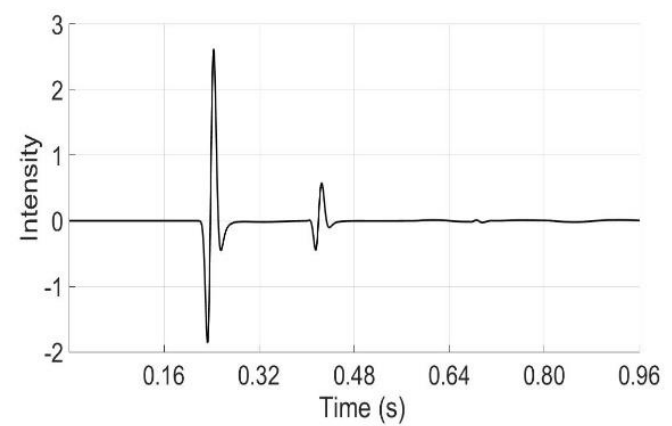

(b)

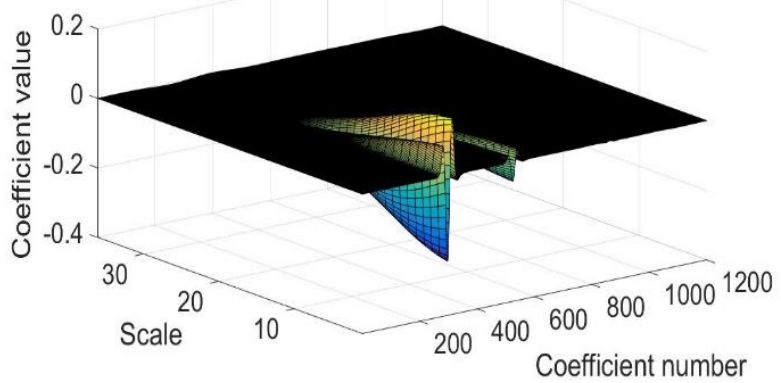

(c)

Figure 3. a) $S_{x_{i}}(z, t)$ wavefield at $x=1.125 \mathrm{Km} \mathrm{b}$ ) Signal $S_{x_{i}, z_{i}}(t)$ at $x=1.125 \mathrm{Km}$ and $z=0.45 \mathrm{Km} \mathrm{c}$ ) Coefficients of CWT of signal in b).

Figure 4 shows the extraction of information of the source wavefield in the downgoing component. Figure 4a) corresponds to the complete source wavefield and Figure $4 b$ ) is the obtained downgoing source wavefield by the algorithm described above. 


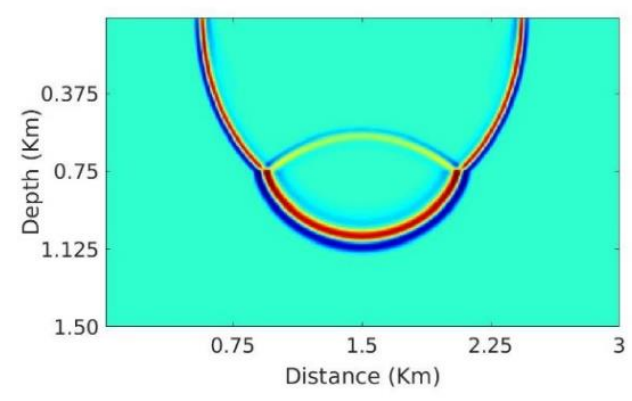

(a)

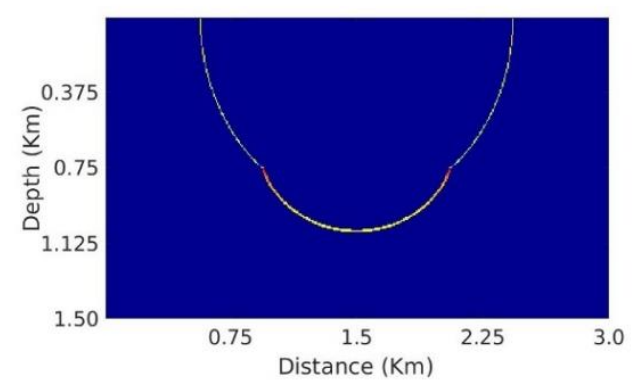

(b)

Figure 4. Extraction of information of downgoing source wavefield of the two-layer velocity model. a) $S(x, z, t)$ when $t=0.36 \mathrm{~s} \mathrm{~b}) S_{N}(x, z, t)$ when $t=0.36 \mathrm{~s}$.

To analyze the receiver wavefield, with a fixed value of $x=$ $x_{i}$, the $R_{x_{i}}(z, t)$ wavefield is obtained from the $R(x, z, t)$ wavefield. Figure () shows the $R_{x_{i}}(z, t)$ wavefield from an arbitrarily chosen value of $x$.

For each value in $t=t_{i}$ axis, the 1D-CWT is performed on the $R_{x_{i}, t_{i}}(\mathrm{z})$ wavefield. Figure $\left.5 \mathrm{a}\right)$ shows a $R_{x_{i}}(z, t)$ wavefield at $x=1.125 \mathrm{Km}$ and, Figure $5 \mathrm{~b}$ ) and $5 \mathrm{c}$ ) show the signal $R_{x_{i}, t_{i}}(\mathrm{z})$ at $t=0.48 \mathrm{~s}$ and its corresponding coefficients obtained by CWT of $R_{x_{i}, t_{i}}(\mathrm{z})$.

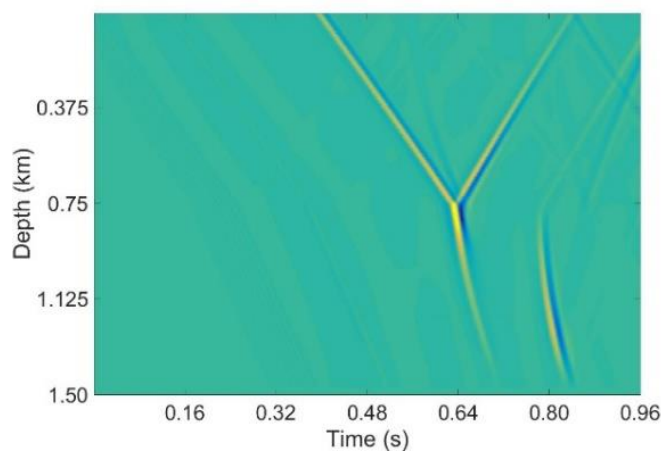

(a)

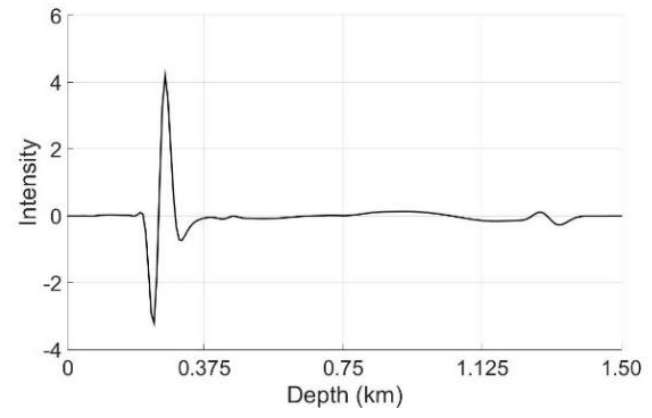

(b)

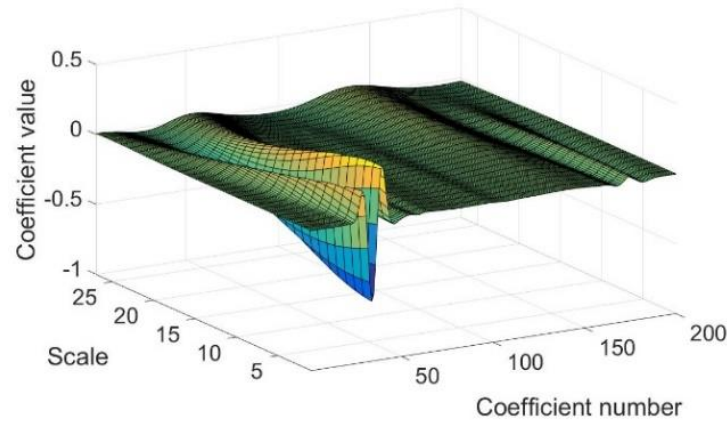

(c)

Figure 5. a) $R_{x_{i}}(z, t)$ wavefield at $\left.x=1.125 \mathrm{Km} \mathrm{b}\right)$ Signal $R_{x_{i}, t_{i}}(z)$ at $x=1.125 \mathrm{Km}$ and $t=0.48 \mathrm{~s} \mathrm{c}$ ) Coefficients of CWT of signal in b).

Figure 6 shows the extraction of information of the receiver wavefield in the upgoing component. Figure 6a) corresponds to the complete source wavefield and Figure $6 b)$ is the obtained upgoing receiver wavefield by the algorithm described above.

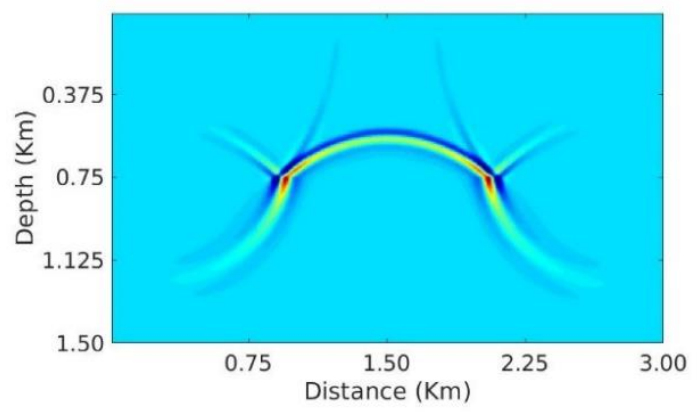

(a) 


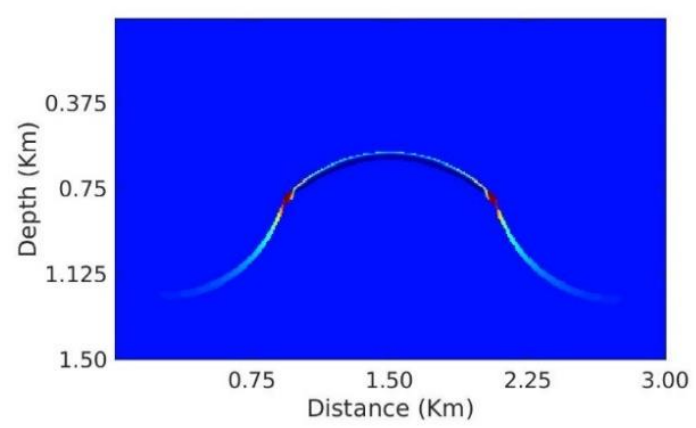

(b)

Figure 6. Extraction of information of upgoing receiver wavefield of the two-layer velocity model. a) $R(x, z, t)$ when $t=0.36 \mathrm{~s}$ b) $R_{N}(x, z, t)$ when $t=0.36 \mathrm{~s}$.

With $S_{N}(x, z, t)$ and $R_{N}(x, z, t)$ wavefields obtained by using this methodology, we use eq. (8) to obtain the migrated image.

Figure 7 shows the migrated image of the velocity model in Figure 1 using the proposed method. As it can be seen the migration noises (artifacts) present in Figure 1 are removed

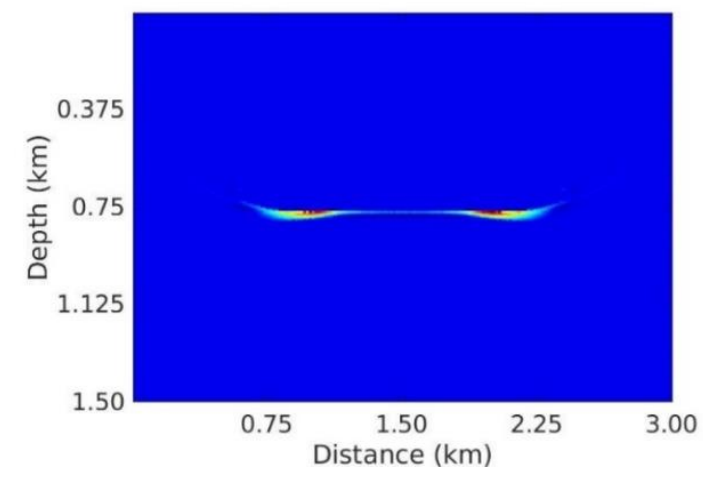

Figure 7. Cross-correlation image of two-layer model with extraction of information of wavefields.

The proposed method is applied to another synthetic dataset. The small salt has a horizontal distance of 1.27 $\mathrm{km}$ and a vertical distance of $0.79 \mathrm{~km}$. We used 3 source points. The first source is located at $x=0.32 \mathrm{~km}$ and the last one at $x=0.95 \mathrm{~km}$ from the beginning of the model; source interval is $315 \mathrm{~m}$; each source point contains 400 receivers and receiver interval is $3.75 \mathrm{~m}$.

When we applied the methodology to more complex models, the downgoing component of the source wavefield can be extracted adequately. The extraction of data related to the upgoing component of the receiver wavefield was not achieved. However, we use only the data extracted from the source wavefield, that is, the downgoing component of the source wavefield, and it is correlated with the complete receiver wavefield, and it is being studied and features obtained by CWT are being analyzed.

The seismic migrated field is improved in comparison with the conventional seismic migrated field obtained with the conventional zero-lag cross-correlation imaging condition.
Figure 8 shows the velocity model and the migrated image using the conventional zero-lag cross-correlation of the small salt model.

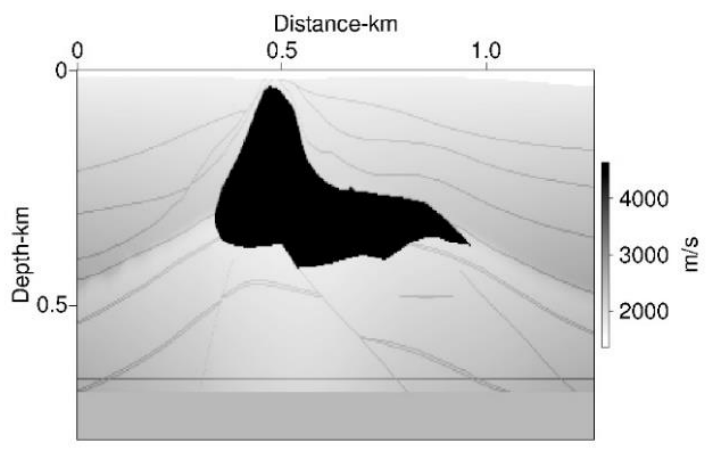

(a)

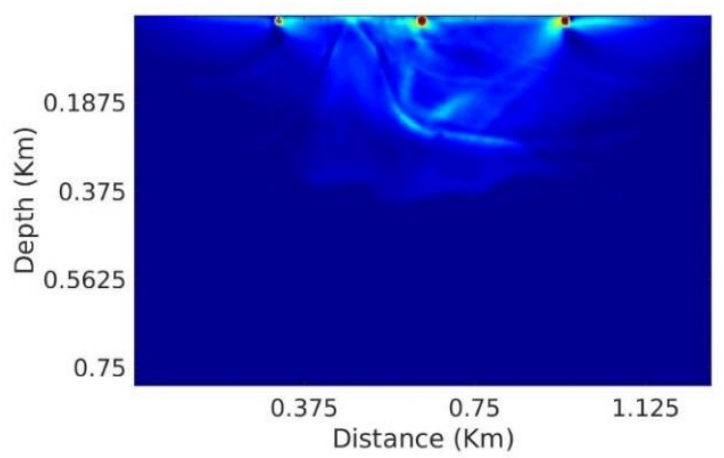

(b)

Figure 8. (a) Small salt velocity model, and (b) migrated image with the conventional zero-lag cross-correlation imaging condition.

Figure 9 shows a comparison between images obtained by using a conventional cross-correlation imaging condition (Figure 9a)) and the proposed method with the crosscorrelation between $S_{N}(x, z, t)$ and $R(x, z, t)$ wavefields (Figure 9b)).

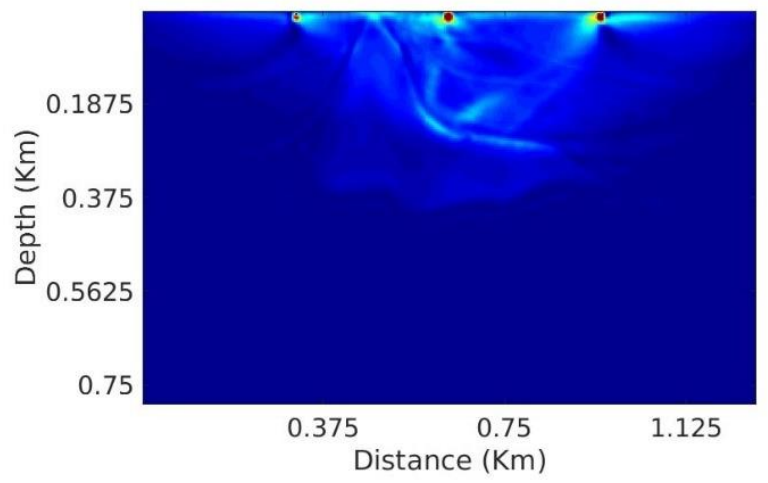

(a) 


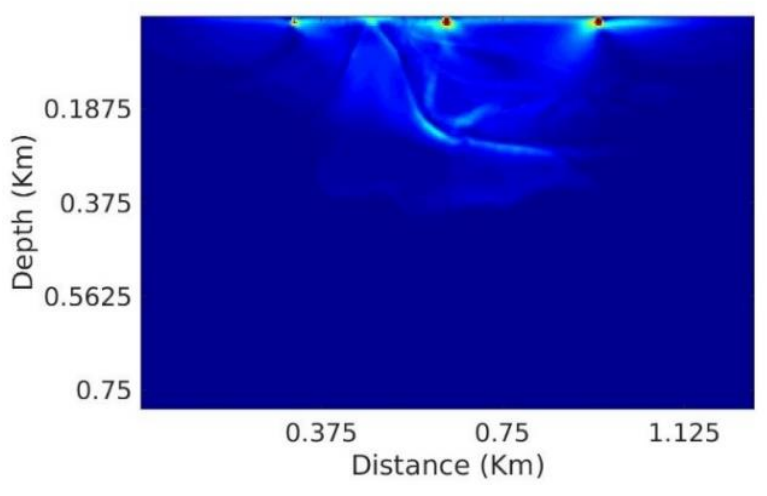

(b)

Figure 9. Comparison of migration results of the small salt model using a) the conventional cross-correlation imaging condition b) the proposed method using only the separated source wavefield.

We can be noted that the artifacts are removed in some regions and the migrated image is improved. Lowfrequency artifacts are reduced in shallow parts of the image and close to flanks of the salt body

\section{Conclusions}

we proposed the use of the CWT to extract relevant information about the source and receiver wavefields in order to extract some parts of components of these wavefields. According to the similarity of the characteristics of scalograms, the values of the CWT coefficients located in the time-scale domain were located in the time-space domain, finding the values of a relative maximum and its neighborhood, both for source and receiver wavefields. This allowed us to locate the reflective events with a better accuracy and obtain an improved migrated image, with an evident reduction of artifacts. This method was applied in simple and complex velocity models and results were promising. A modification of the conventional zero-lag cross-correlation imaging condition was used. This modified imaging condition is the same that is used in oneway wave equation methods. We achieved the improvement of the image using only the separated source wavefield and the complete receiver wavefield, considerably reducing artifacts induced by the undesired correlation of some subsets of source and receiver wavefields. The information about the downgoing component of the source wavefield can be extracted adequately. The information about the upgoing component of the receiver wavefield was partially extracted and in future work, the full extraction of information about this component of the receiver wavefield will be studied and the characteristics obtained through the CWT and Wavelet transform Modulus Maxima (WTMM) will be analyzed.

\section{Acknowledgments}

We would like to express our gratitude with Colombian Oil Company ECOPETROL and COLCIENCIAS for supporting this work as a part of the research project grant No. 0266-2013

\section{References}

BAYSAL, E., KOSLOFF, D. D. \& SHERWOOD, J. W. A two way nonreflecting wave equation, Geophysics, 49, 132 1984.

CHATTOPADHYAY $S$ \& MCMECHAN G. Imaging conditions for prestack reverse time migration. Geophysics, 73 (3), p. S81-S89, 2008.

CHEN, T. \& HE, B. A normalized wavefield separation cross-correlation imaging condition for reverse time migration based on Poynting vector, Applied Geophysics, 11, p. 158-166, 2014.

CLAERBOUT, J.F. Toward a unified theory of reflector mapping. Geophysics, 36, p. 467-481, 1971.

CLAERBOUT, J.F. Imaging the Earth's interior, Blackwell Scientific Publications, California, 398 pp, 1985.

FEI, T. W. \& LUO, Y. De-blending reverse time migration, SEG 2010 International Exposition and Annual Meeting, p. 3130-3134, 2010.

GUITTON, A., KAELIN, B. \& BIONDI, B. Least-square attenuation of reverse time migration Geophysics, 72 (1), p. S19-S23, 2007.

GUITTON, A., VALENCIANO, A., BEVC, D. \& CLAERBOUT, J. F. Smoothing imaging condition for shotprofile migration, Geophysics, 72, p. S149-S154, 2007.

KAELIN, B. \& GUITTON, A. Imaging condition for reverse time migration, $76^{\text {th }}$ International Annual Meeting and exposition, SEG, Expanded abstracts, p. 2594-2598, 2006.

LIU F, ZHANG, G., MORTON S \& LEVEILLE J. An effective imaging condition for reverse time migration using wavefield decomposition. Geophysics, 76, p. 10-29, 2011.

LOEWENTHAL, D., STOFFA, P. \& FARIA, E. Suppressing the unwanted reflections of the full wave equation, Geophysics, 52, p. 1007-1012, 1987.

PANIAGUA, J. G., SIERRA-SOSA, D. \& QUINTERO, O. $\mathrm{L}$. Laguerre-Gauss filters in reverse time migration image reconstruction, Revista Brasileira de Geofísica, 35 (1), p. 1-13, 2017.

PANIAGUA, J. G, Improving the seismic image in Reverse time migration by analysis of wavefields via continuous wavelet transform. PhD thesis, School of Sciences, EAFIT University, Medellín, Colombia.

PESTANA, R., DOS SANTOS, A. \& ARAUJO, E. RTM imaging condition using impedance sensitivity kernel combinated with the Poynting vector, SEG Technical Program Expanded Abstracts, p. 3763-3768, 2014.

SCHUSTER, G. T. Basics of seismic imaging, Cambridge University Press, 137 pp 2010.

VALENCIANO, A. \& BIONDI, B. 2-D Deconvolution imaging condition for shot profile migration, 73th International Annual Meeting and exposition, SEG, Expanded abstracts, p. 1059-1062, 2003.

YOUN, O. \& ZHOU, H. Depth imaging with multiples, Geophysics, 66, 246-255, 2001. 